\title{
Detecting and predicting the impact of land use changes on groundwater quality, a case study in Northern Kelantan, Malaysia
}

\begin{abstract}
The conversions of forests and grass land to urban and farmland has exerted significant changes on terrestrial ecosystems. However, quantifying how these changes can affect the quality of water resources is still a challenge for hydrologists. Nitrate concentrations can be applied as an indicator to trace the link between land use changes and groundwater quality due to their solubility and easy transport from their source to the groundwater. In this study, 25 year records (from 1989 to 2014) of nitrate concentrations are applied to show the impact of land use changes on the quality of groundwater in Northern Kelantan, Malaysia, where large scale deforestation in recent decades has occurred. The results from the integration of time series analysis and geospatial modelling revealed that nitrate (NO3-N) concentrations significantly increased with approximately $8.1 \%$ and $3.89 \%$ annually in agricultural and residential wells, respectively, over 25 years. In 1989 only $1 \%$ of the total area had a nitrate value greater than $10 \mathrm{mg} / \mathrm{L}$; and this value increased sharply to $48 \%$ by 2014 . The significant increase in nitrate was only observed in a shallow aquifer with a $3.74 \%$ annual nitrate increase. Based on the result of the Autoregressive Integrated Moving Average (ARIMA) model the nitrate contamination is expected to continue to rise by about $2.64 \%$ and $3.9 \%$ annually until 2030 in agricultural and residential areas. The present study develops techniques for detecting and predicting the impact of land use changes on environmental parameters as an essential step in land and water resource management strategy development.
\end{abstract}

Keyword: Land use changes; Deforestation; Nitrate; Geospatial modeling; ARIMA model 